\title{
New Trends in Medical Education During and Post COVID-19 Pandemic
}

\author{
Pushpa Potaliya and Surajit Ghatak
}

\begin{abstract}
This sudden emerged crisis of COVID-19 pandemic has forced the medical units globally with practical and logistical trial and apprehensions for patient wellbeing. In addition, it has affected medical education by forcing them to stay away from the institutes for preventing potential spread of virus. Mean time conventional classroom teaching and inhand experience in training are affected. This short communication discusses the current transformation in medical education by adoption of newer trends, describes how COVID-19 may affect the standard curriculum and learning milieus, and scouts impending consequences in Post COVID19 era for the future of medical education.
\end{abstract}

\section{Index Terms-COVID-19; Education; Medical; trends}

\section{INTRODUCTION}

Living through these unprecedented period calls for ardent contemplation of significant and existential difficulties. Even though the obligatory emphasis has been on patient care and community well-being, the emergence of pandemic due to SARS COV-2 has disrupted medical education and need resilient and rapid feat from policy makers and medical educators. A pneumonia of unknown cause was detected in Wuhan, China and first reported to the WHO Country office in China in December 2019. In the end of January 2020, the outbreak was declared a public health emergency of international concern. On 11 February 2020, WHO announced the name 'COVID-19' for the novel coronavirus disease [1]. The global pandemic is like a wave- one that is crashing through all continents, symptoms ranging from none to all, to deadly and killing thousands. Deadly virus involves a spreading problem as it is constantly mutating. Major ways to flatten the curve is widespread vaccination and developing herd immunity by widespread infection that lead to immunity. Both of this are months or may be years away and meantime havoc is reigning.

Combating this global health crisis involves various strategies, moving from containment to mitigation and the need for social distancing. The crucial and long-term necessity is learning to consciously live with the disease. A non-pharmaceutical infection and control intervention named 'Social/physical distancing' is globally accepted key component of recommendations to moderate the community spread. After early blinding from the jeopardy of the pandemic by global leadership, measure of practicing social

Published on May 30, 2020.

P. Potaliya, All India Institute of Medical Sciences Jodhpur, India. (corresponding e-mail: ppotaliyaa@gmail.com)

S. Ghatak, All India Institute of Medical Sciences Jodhpur, India.

(e-mail: surajit_ghatak@rediffmail.com) distancing ranging from voluntary to enforce various degrees of lockdown accelerated quickly. Governments announced states of emergency, travel restrictions become multifarious or even complete restriction in red zones, and educational institutions from school to universities declared closed. In educational scenario, these tactical defensive measures made the necessity of adapting novel virtual teaching-learning methods inevitable. In terms of medical education, however, various other multidimensional intricacies are also encountered. The configuration of medical education in India chiefly gyrates around two practices that is between basic instruction in the classroom and patient-centered teaching in the clinic. In the context of physical presence in classroom and patient-centered teaching, current mandate to prolapse the curve, prior to reforming medical education, many queries need to be address. How the future doctors be trained within the limitations of social distancing? What should be the competencies in curriculum? What about the in-hand experience of practical work in labs? Subsequent to webbased teaching-learning and furnishing learning resources and digital content, is it possible to simulate virtual patient interface?

It is already more than a 5-week suspension on for the medical students' from participating in any physical presence in classroom and practical laboratory sessions. Based on operational feasibilities individual schools will further decide on the duration of suspension. In the current advanced digital world besides online classes, this interlude is compelling educators on compilation and broadcasting of information and resources and to exercise and adapt other alternative teaching- learning approaches. Even experts on recent development in present time opined the reconfiguration of medical education inevitable. They stated that to accomplish it, fueling has to be done by incorporating technology through online educational platforms and the requisite to transform clinical training to more outpatient setups with competency based promotion rather than time [2].

\section{A. End of conventional classroom instruction}

As soon as online education seasoned into a standard model, the configuration of the preclinical classrooms will also show transformation. Sixty minute/ two hours didactic presentations will not be favored in online teaching learning. As a substitute, a series of short i.e. about 6 to 15 minutes video sessions, each covering not more than couple of main points supplemented with required text and link of reference resources is considered appropriate [3]. When the medical curriculum will be designed and recorded with the preliminary objective of been utilized online, the short sessions including clinical case scenarios or illustrations, demonstrations on specimen or on virtual software's with relevant text could become the gold-standard. 


\section{B. Remote modes of Learning}

To achieve quality learning in testing time as COVID-19 crisis, remote learning is a worldwide accepted approach. It requires institutions to organize themselves for multi-modal responses, utilizing existing infrastructure to its best and use a blend of different learning medium to warrant that the students are engaged and learning.

General Principal for remote learning strategies:

\section{A. Planning}

i. Develop short and long-term plan: Depending upon organizations capacity and existing capitals a multifaceted model incorporated with amalgamation of technology and its delivery mechanisms are planned. Short-term plan focuses on selected topics and is time bound until the classrooms come re-instate. Long-term comprises of contract out stakeholders as startups, technology companies, broadcasters etc. to upgrade selected modality.

ii. Emphasis on curating existing content (available open access or free) and aligning it in accordance to curriculum. An inventory can be made to be used by means of remote learning.

iii. Content has to be strictly in accordance with curriculum to fulfill learning objective.

iv. Virtual support system for learners and facilitators both has to be created.

B. Mitigation and execution of remote learning

C. Data dispensing

Various models are there for implementing education through remote learning. It comprises of:

A. Online remote learning model

1. Develop institutional Learning Management System as centralized online platform to deliver all accessible content at one place. It shall include content, tools, resource material, links, etc. It should support varied operating systems and software applications and can be accessed by extensive potential user base and multiple devices as mobile, tablets, laptops etc. Online learning prospects should be obligatory to be accessible through low bandwidth and miserable latency state.

2. Short videos of live classes or pre-recorded lectures are best way to cover the topics. Here the key for optimization is short duration of videos for better understanding.

3. Utilizing Technology

These days several education and technology clusters and interactive streaming arenas are joining forces to provide or create rapid and easily accessible digital platforms in least of possible duration for sustained education. For this, these enterprises are offering tools free of cost for certain duration and/or at nominal subscription charges later [4]. Institutions utilize one or another among them as per their requirement.

i. One of most extensively used among these is Google. It is providing unrestricted access to its basic features with no charges. For the advanced ones it has a monthly subscription charges as for Hangouts Meet to all G Suite and G Suite Education clients.

ii. 'Microsoft' is another giant offering a limited duration free trial at various countries of its highest tier of Microsoft Teams to enable institutions to function swiftly. iii. One most talked about provider is 'Zoom' which took a massive popularity in this crisis period. It raised controversies too but company is rolling newer versions to overcome the issues encountered by users.

iv. Remote teaching learning gets simpler and widely accessible by cloud meeting and team collaboration tools such as Tencent's WeChat Work or Alibaba-owned DingTalk.

v. Video conferencing tools as GoToMeeting and GoToWebinar of 'LogMeIn' is offering organizations access to its tools and utilized broadly.

vi. Webex tool by 'Cisco' is invigorating to suitably assist institutions round the clock in more than forty countries.

Online education modules can also provide platform for interactive sessions such as synchronous problem solving sessions with subject expertise as well as peer discussion forums. These classes will necessarily a comprehensive learning experience in practical aspect, armed with more inclusivity, consistent technology and, most significantly, artificial intelligence (A.I) based augmented reality and virtual reality. Eventually such virtual session imparts effect almost analogous to discussion in classroom. For example, in anatomy, histology lectures and microscopic pictures of slides is a digitally shared resource but augmented with digital simulations for existing dissection will be improved with virtual reality.

\section{Offline remote learning model}

Textbooks (also in pdf formats), study material, work sheets, resource and reference material can be disseminated electronically utilized in technology compromised set ups.

5. Through educational television:

Several countries have used this for decades. One prominent examples Mexico. Telesecundaria is oldest example of educational television in latin America dating back to 1968 and led to formation of educational television network [5]. In this, three main programs are possible: Live broadcast, pre-recorded broadcast and edutainment programs. Important points to establish it are:

1. Curating content

Focus on curating content beyond short-term pandemic duration. Also, content broadcasted can have subtitles/language options to enhance its accessibility globally. It can be done as:

i. Live broadcast: It can be done by instructor in staged classroom setting and broadcasting recorded session on television. These are lowcost rapid production

ii. Pre-recorded broadcasts: Broadcasts are done of high demand or maximum viewed online content. Here these contents are to be purchased from private owners or developers. Compromising the video quality, Licensing and copyright issues are certain drawback associated with it.

iii. Edutainment series: Here the topics are taught using comic strips, plays, crosswords, acronyms, puzzles, recorded quizlets etc.

2. Broadcasting sources/channels 
Private broadcasters or institutional network can be developed. Sponsors can provide financial support based on restricted advertisement of products.

3. Communication strategy

Launch of program can be intimated by phones, mails or via instant messenger groups. Schedule can be delivered in advance or time to time by intimation.

4. Helpdesk/support and feedback

A toll free number, text messages, dedicated email, social media links can be provided for FAQ'S and technical hitches. Even feedback can be collected and assessed for each session by it.

6. Through educational radio

It can be utilized best for two-way interaction in doubt solving, feedback or assessing through quizzes on this platform. This is of great benefit at regions where internet connectivity is an issue and radio stations exist.

The shift to digital education was undoubtedly arbitrary, as it was unintended for this time but such learning in such crisis will enable the educators and educational institutions to be implemented in the future.

\section{Fast-track Transformation in Medical Education}

To enable the development of establishing futuristic medical schools by fast-track transformation of current ones, the central/nationalized funding agencies are required to provide more grants. These would be helpful in establishment and to uphold the medical education in accordance with curriculum. Also, it can help in delivering trainings for proficiency with the altering needs of health care sector. Following steps has to be taken care of:

1. Develop novel technology modes and tools for evaluating and assessing imperative competencies for medical students at different training stages to form personalized learning modules.

2. Plan the modules to support quick innovation among the schools and to disseminate the ideas being tested to additional medical schools.

3. To create collaborative projects and a common assessment plan so that evidence-based practices are assessed, shared, and executed among various medical schools.

4. The consortium schools can be developed comprising of team members from various medical schools, advisory panel, medical education experts, technoexperts from digital world. They all should work together to align recent recommendations on medical school graduation competencies to better define assessment strategies.

5. A core set of material is ear-marked for the first phase of medical education, with multiple diverging opportunities for individualized study followed by expertise in it.

6. Comprehensive electronic portfolio systems linked to learning management systems have to be developed, and novel roles for faculty trainers should be done.

7. Enhancing learning through utilization of technology can be a core theme at many schools which are currently deprived of it.

\section{Challenges and solutions}

Technology complications encountered by facilitators and learners is a huge challenge to overcome. Moreover, when diligently structured with relevant collaborative assignments or discussion groups, online modules can allow and establish the development of profound, shared learning networks between students. Imperative measures can be devised as for example, at the commencement of online program; a couple of sessions for accustoming learners can be deliberated. These has to be interactive so as it solve all the queries of students and instill sense of team amongst them. Helpdesk and feedback are other supporting measures that can aid in resolving challenges and improve the designed system.

Another ambiguity is that medical education completely online is challenging. Partially it is because online modules are presently offering content to cover up the course as per schedule and on time. Mere finishing the course without proper understanding is inadequate for medical curriculum. However, in global crisis or unprecedented times when not many options are available with institutions and students have semester credits at stake, relying on online modules is must. Such modules just requires tangible enthusiasm and perseverance to learn [6].

Uncertainty is also concerning the duration of crisis and cumulative future apprehensions for more waves until resuming the usual 'Normal' back. Here the challenge is to offer authentic patient experiences for students as it is a vital element of medical education and difficult to carry out in present COVID-19 scenario.

\section{CONCLUSION}

\section{$A$ vision for futuristic medical schools}

Futuristic Medical schools will be the organizations wellprepared and compliant for prompt transformation i.e. whether in technology, in broadcasting, data propagation, information dispensing, in tele communication. Meanwhile there responsibility is also in conditioning the settings that are accountable for fostering clinicians to be constant learners throughout life. They should be capable in utilization of new facts and figures, information, skills, data, webinars and technologies efficiently as constructive gears for finest patient care in clinical set-up [7].

Also, why to restrain resources and material confined to a particular set of students? On being available online other associated dynamic cannot be neglected. So open and easy accessibility is to be kept in mind for future as it enables students to learn from the best expertise of the field globally. Collectively when whole world is fighting in this pandemic for best solutions for mankind, developing the novel models for medical schools that will efficiently enable the futuristic clinicians to meticulously do patientcare and serve the communities in their best capacity. The COVID-19 pandemic seems to be a crucial turn that is compelling us to transform ways in teaching learning medicine. In the inevitable transformation that has already started a creative thinking is required. What lies in future no one knows and only future will state if the disruption will be ephemeral or permanent but to ensure the sustainability of medical education - we should consider transformation from now. 


\section{REFERENCES}

[1] COVID-19 updates and resources. Liaison Committee on Medical Education, Updated March 25, 2020, Accessed March 27, 2020.

[2] E. J. Emanuel, "The inevitable reimagining of medical education," $J A M A, 2020$ February 27. "to be published"

[3] R. Berg, A. Brand, J. Grant, J.S. Kirk, and T. Zimmerman, "Leveraging recorded mini-lectures to increase student learning. Course Design", Course design, vol. 14(2), pp. 5-8, 2014.

[4] S. McQuaide, "Making Education Equitable in Rural China through Distance Learning”, Int Rev Res Op Dist Learn, vol. 10(1), pp.13940, 2009.

[5] J. Moran, G. Briscoe, S. Peglow, "Current Technology in Advancing Medical Education: Perspectives for Learning and Providing Care", Acad Psychiatry, vol. 42, pp. 796-99, 2018.

[6] C. Hart, "Factors associated with student persistence in an online program of study: a review of the literature", J Interact Online Learn, vol. 11(1), pp.19-42, 2012.

[7] S. E. Skochelak, S. J. Stack, "Creating the medical schools of the future", Acad Med, vol.92(1), pp. 16-19, 2017.

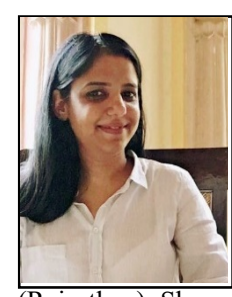

Potaliya P. Jodhpur (Rajasthan), INDIA. Date of Birth: 23 May 1983. Educational background includes Ph.D in anatomy in year 2011 from Dr.S.N.Medical College, Jodhpur (Rajasthan) in India. Field of study is Human Anatomy.

She is having experience of around 13 years in field of teaching and research at various renowned medical institutions. Earlier she worked as assistant professor at Dr.S.N.Medical college, Jodhpur (Rajasthan). She completed her senior residency from All India Institute of Medical Sciences, Jodhpur from 2014-17 and joined as permanent faculty on the post of assistant professor here only in 2017. She has authored more than fifteen publications in various national and international journals. She also has authored a book on Gross anatomy titled 'Salubris Prep manual anatomy vol 3(Head and neck), Jodhpur, Rajasthan, Salubris Publishers, 2020'. She has worked on gross anatomy, histology, immunohistochemistry and currently working in tissue culture. and the other authors may include biographies at the end of regular papers Dr.Potaliya is presently member of various national and international societies as Royal college of Biology, American association for Anatomy, life member of Anatomical society of India etc. In 2018 she was awarded with best paper presentation at an international conference on Genetics at Puducherry, India. 